\title{
ORT_21 - SARS-CoV-2 inactivation strategies for safe use in diagnostic and research
}

José Henrique Rezende Linhares ${ }^{1 *}$; Mariana Pierre de Barros Gomes ${ }^{1}$; Ygara da Silva Mendes ${ }^{1}$; Tiago Pereira dos Santos ${ }^{1}$; Juliana Fernandes Amorim da Silva ${ }^{1}$; Viviane Silva Gomes ${ }^{1}$; Debora Ferreira Barreto Vieira $^{2}$; Marcos Alexandre Nunes da Silva ${ }^{2}$; Marta Cristina de Oliveira Souza'; Sheila Maria Barbosa de Lima ${ }^{1}$.

${ }^{1}$ Fiocruz/Bio-Manguinhos;

${ }^{2}$ Fiocruz/IOC.

Introduction: The expanded interest in studying SARS-CoV-2 to address the current pandemic requires that many laboratories acquire the capacity to work with the virus. However, safety is one of the main limiting factors in a SARS-CoV-2 study due to the high risk of transmission and exposure of healthcare professionals and scientists. Therefore, the infectious virus must be handled in a BSL3 laboratory or higher and it is necessary the development of methods to safely inactivate the virus and to allow a set of studies to be carried out at lower levels of biocontainment. Successful inactivation of the virus allows the material transfer from a BSL3 to a BSL2 environment, enabling its safe use in applications such as standards to challenge diagnostic kits, ELISA and development of monoclonal antibodies.

Objective: In this study, different methodologies for inactivation of SARS-CoV-2 were evaluated in order to produce a batch of inactivated viruses. The criteria for selecting the best condition(s) include: inactivation capacity greater than $99.9 \%$ of the virus; cost, execution time, scale up capacity and integrity of the viral particle and genome.

Methodology: 44 different conditions were tested between chemical and physical agents (Ascorbic acid, Guanidine, Glutaraldehyde, Beta-propiolactone-BPL, and high temperatures). For screening, $\mathrm{TCID}_{50}$ and RT-qPCR assays were performed to assess the inactivation profile and the maintenance of SARS-CoV-2 RNA copies, respectively. The best results were subjected to new screening, including Transmission Electron Microscopy (MET) and serial passages to ensure viral inactivation. Complete inactivation was indicated by absence of CPE in all sub cultured flasks and by quantifying the absence of viral replication by qPCR in the culture supernatants.

Results: The chemical agents BPL, Glutaraldehyde and Guanidine showed equivalent inactivation efficiency (> 99.99\%) compared to Ascorbic acid (70\%). The detection of viral RNA by RT-qPCR showed that BPL and Guanidine were more efficient in maintaining RNA quantification $(<0.5 \mathrm{Log})$, when compared to Glutaraldehyde $(>1 \mathrm{Log})$. MET images suggest that BPL was the only chemical agent to preserve the structure of the viral particle, which is an important feature for selecting the inactivation methodology. Temperature inactivation is apparently dependent on the sample volume and makes it difficult to scale up the process.

Conclusion: After the characterization steps, BPL inactivation was selected for the production of the inactivated SARS-CoV-2 bank, which will serve as an input to assist LATEV partners in diagnostic tests and quality control of Bio-Manguinhos kits, in addition to attending tests functional for the detection and selection of antibodies. The next step is to establish methodologies for purifying inactivated material to increase the specificity of antibody selection and recognition.

Keywords: SARS-CoV-2; Inactivation; Covid-19 